\section{Supporting the urban poor to quit tobacco: adding years to lives}

\author{
Kamran Siddiqi, ${ }^{1}$ Helen Elsey ${ }^{2}$
}

Tobacco use is in decline in most highincome countries (HICs); however, the number of tobacco users are rising in many low/middle-income countries (LMICs). ${ }^{1}$ Consequently, by 2030 tobacco-attributable deaths are projected to rise to 8.3 million per year with more than $80 \%$ occurring in LMICs. ${ }^{1}$ Furthermore, the tobacco-attributable burden is not evenly distributed within these nations; being highest in socioeconomically disadvantaged and marginalised sections of society. ${ }^{2}$ The urban poorthe participants of the trial published by Sarkar $e \mathrm{al}^{3}$ in Thorax-are particularly vulnerable. For example, $55 \%$ of the poorest 20th centile of urban male residents in South Asia use tobacco in contrast to $40 \%$ of the richest. ${ }^{4}$ Quitting tobacco leads to immediate benefits and if stopped before the age of 40 , the associated risk of death is reduced by $90 \% .^{5}$ However, tobacco being an addictive substance, most users struggle to quit and many only do so with behavioural and/or pharmacological support. ${ }^{6}$ According to Global Adult Tobacco Survey, more than $30 \%$ of smokers in LMICs attempted to quit in the previous year (15\% in China). ${ }^{7}$ However, in most countries in particular LMICs, cessation advice or services are not available. ${ }^{8}$ Even where they are, the urban poor are least likely to receive cessation advice through traditional routes because of limited access to affordable healthcare. ${ }^{9}$

Sarkar $e t a l^{3}$ present the findings of the Babex trial-a randomised controlled trial designed to assess the effectiveness of a brief community outreach intervention on tobacco cessation in the urban poor. The investigators randomised 32 residential localities within some of the most impoverished areas of Delhi, India, and allocated these to two arms. Adult tobacco users received outreach interventions in the community. Those in the intervention arm received quit advice and training in yogic breathing exercises delivered in a

${ }^{1}$ Department of Health Sciences, University of York, York, UK; ${ }^{2}$ Leeds Institute of Health Sciences, University of Leeds, Leeds, UK

Correspondence to Dr Kamran Siddiqi, Department of Health Sciences, University of York, Seebohm Rowntree Building, Heslighton, York Y010 5DD, UK; kamran.siddiqi@york.ac.uk single session of approximately $15 \mathrm{~min}$ duration; those in the control arm received very brief quit advice lasting for less than $1 \mathrm{~min}$. Researchers independent of the interventions, conducted follow-up assessments of smoking status at 4 weeks and 7 months. Self-reported quits were biochemically verified through salivary cotinine testing. Eighty per cent of the 1213 participants were male. More than $95 \%$ completed follow-up and at 6 months, $2.6 \%(16 / 611)$ of participants were found to be continuously abstinent in the intervention arm compared with $0.5 \%(3 / 602)$ in the control arm. The effect, though modest, was statistically significant. The study did not suggest any difference in the quit rates between those that used smoking and smokeless forms of tobacco.

This is a well-designed study that recruited a large sample of participants, targeted marginalised sections of the society, included all forms of tobacco users, tested a culturally adapted but theory-driven intervention and verified all self-reported abstinence reports biochemically. The investigators also achieved impressive trial recruitment and retention rates despite recruiting within socioeconomically disadvantaged communities. However, there are some study limitations. A behavioural intervention is dependent on a number of contextual factors. These include target behaviour, participants' attributes, contents of the intervention, providers' attributes and approach to intervention delivery. ${ }^{10}$ Therefore, trials of behavioural interventions need to recruit a range of providers to enhance external validity of their findings. The Babex trial involved only two providers-a physician and a community health worker. The authors' claim that this was a non-physician dependent intervention is also questionable. The authors stated that the intervention is potentially cost-effective. A comprehensive economic evaluation will be needed to establish this. Suggesting that the intervention is potentially scalable is sensible. However, further implementation research is needed to explore how to optimise this and ensure sustainability. Finally, the authors are correct in comparing this brief community outreach intervention with many other low-intensity smoking cessation interventions such as brief advice delivered by a physician or single-session individual behavioural support. Predictably, the intervention effect observed in the Babex trial, though modest, is also comparable with that observed in other trials of brief physician-based advice ${ }^{11}$ and individual behavioural counselling. ${ }^{12}$ However, it is debateable whether it can be presented as a substitute to more effective cessation interventions. An alternative position would be to always advocate for the most cost-effective treatments as first line and only resort to less effective but low-cost options when these are not available.

What is remarkable about the Babex trial is that the intervention was found to be effective in the urban poor. Previously, most trials have struggled to show an effect of smoking cessation interventions in disadvantaged populations unless they have used tailor-made interventions. ${ }^{13}$ This could explain the findings of the Babex trial where the intervention was culturally adapted to cater for tobacco users living in the urban slums of Delhi. The other population-based approaches, which achieve comparable effect size, include telephonebased counselling (eg, quit lines), ${ }^{14}$ mobile phone-based interventions (eg, text messages) ${ }^{15}$ and internet-based counselling. ${ }^{16}$ Access to such interventions is likely to improve in LMIC due to wider availability of the internet, mobile phones and smartphones. Future research should compare the effect of such digital interventions with more traditional face-to-face approaches to cessation in socially disadvantaged populations.

Article 14 of the WHO Framework Convention on Tobacco Control, makes it obligatory for its signatory countries to offer cessation support. ${ }^{17}$ However, according to a survey of tobacco cessation services conducted in 121 countries, most LMICs have not made this a priority. ${ }^{8}$ Only a handful of countries offer telephone-based counselling or any specialist behavioural support. ${ }^{8}$ Cost considerations are often a barrier and therefore potentially low-cost interventions such as the one used in the Babex trial can have a big population impact in countries dealing with large number of tobacco users. As part of implementing WHO's MPOWER strategy for controlling tobacco use, countries should prioritise tobacco cessation and consider using low-cost and population-based approaches. Using implementation science approaches, the research community should demonstrate the cost-effectiveness, scalability and sustainability of such interventions. 
There is now growing evidence that even low-intensity tobacco cessation interventions can be effective in achieving continuous abstinence. This is particularly useful for tobacco control in LMICs where such interventions can be delivered relatively cheaply with the likelihood of a big population-level impact. Governments should focus their efforts on the impoverished tobacco users and prioritise scaling up such low-cost interventions.

Twitter Follow Kamran Siddiqi at @kamsid66

Competing interests None.

Provenance and peer review Commissioned; internally peer reviewed.

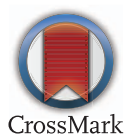

To cite Siddiqi K, Elsey H. Thorax 2017;72:105-106.

Published Online First 4 November 2016

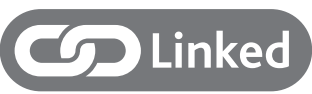

http://dx.doi.org/10.1136/thoraxjnl-2016-208732
Thorax 2017:72:105-106.

doi:10.1136/thoraxjnl-2016-209435

\section{REFERENCES}

1 WHO. WHO global report: Mortality attributable to tobacco. 2012

2 Siddiqi K, Jawad M, Mushtaq N, et al. Tobacco and health disparities. BioMed Res Int 2015;2015:570173.

3 Sarkar BK, West R, Arora M, et al. Effectiveness of a brief community outreach tobacco cessation intervention in India: a cluster-randomised controlled trial (the BABEX Trial). Thorax 2017;72:167-73.

4 WHO. Global report on urban health: equitable, healthier cities for sustainable development, 2016.

5 Jha P, Ramasundarahettige C, Landsman V, et al. 21 st-Century hazards of smoking and benefits of cessation in the United States. N Engl J Med 2013;368:341-50.

6 Stead LF, Koilpillai P, Fanshawe TR, et al. Combined pharmacotherapy and behavioural interventions for smoking cessation. Cochrane Database Syst Rev 2016;3:CD008286.

7 Song Y, Zhao L, Palipudi KM, et al. Tracking MPOWER in 14 countries: results from the Global Adult Tobacco Survey, 2008-2010. Glob Health Promot 2016;23(2 suppl):24-37.

8 Pine-Abata $H$, McNeill $A$, Murray $R$, et al. A survey of tobacco dependence treatment services in 121 countries. Addiction 2013;108:1476-84.

9 Adams AM, Islam R, Ahmed T. Who serves the urban poor? A geospatial and descriptive analysis of health services in slum settlements in Dhaka,
Bangladesh. Health Policy Plan 2015;30(Suppl 1): i32-45.

10 Hiscock R, Murray S, Brose LS, et al. Behavioural therapy for smoking cessation: the effectiveness of different intervention types for disadvantaged and affluent smokers. Addict Behav 2013;38:2787-96.

11 Stead LF, Buitrago D, Preciado N, et al. Physician advice for smoking cessation. Cochrane Database Syst Rev 2013(5):CD000165.

12 Lancaster T, Stead LF. Individual behavioural counselling for smoking cessation. Cochrane Database Syst Rev 2005(2):CD001292.

13 Hill S, Amos A, Clifford D, et al. Impact of tobacco control interventions on socioeconomic inequalities in smoking: review of the evidence. Tobacco Control 2014;23:e89-97.

14 Stead LF, Hartmann-Boyce J, Perera R, et al. Telephone counselling for smoking cessation. Cochrane Database Syst Rev 2013(8): CD002850.

15 Whittaker R, McRobbie H, Bullen C, et al. Mobile phone-based interventions for smoking cessation. Cochrane Database Syst Rev 2016(4): CD006611.

16 Siddiqi K, Dogar O. Can the internet help economically disadvantaged smokers? Lancet Resp Med 2014;2:947-8.

17 Yach D. WHO framework convention on tobacco control. Lancet 2003;361:611-12. 